\title{
Pupil Motility in Long-term Diabetes
}

\section{Dear Sir,}

Our thanks to Dr. Bryant for his interest in the article, "Pupil Motility in Long-term Diabetes" [1]. We are glad that he agrees with the important conclusion of the article, namely that the abnormalities of the long-term diabetic pupil are not generally due to intrinsic "stiffness" of the iris tissue, but are the reflection of autonomic (sympathetic) nervous involvement.

Regarding Dr. Bryant's treatment of the statistics we agree with his own words that 'tests of "significance" would be axiomatically meaningless' regarding interrelationships of sex, age and duration of diabetes in a sample of 8 females and 6 males, all aged 30 to 40 years and with duration of diabetes above 15 years, as is the inference from the a posteriori selected measures of association between ripples on the surface of the n-dimensional data space in the finite sample.

In all cases, with acknowledgements to Galileo Galilei, we think we must be careful to stick to the rule that in diabetes research we publish experiments meant for the verification on an a priori formulated hypothesis. A posteriori hypotheses - based on previous knowledge, experiments or randomly encountered data have their proper place in the Introduction in these publications followed by statements like 'In order to elucidate this problem we have reexamined the pupillary response to a rhythmic light stimulus ...' [1].

Yours sincerely

Á. B. Hreidarsson/H. J. G. Gundersen

\section{Reference}

1. Hreidarsson $\AA$ B (1979) Pupil motility in long-term diabetes. Diabetologia 17: 145-150

\author{
Á. B. Hreidarsson \\ Second University Clinic of Internal Medicine \\ Kommunehospitalet \\ DK-8000 Aarhus C \\ Denmark
}

